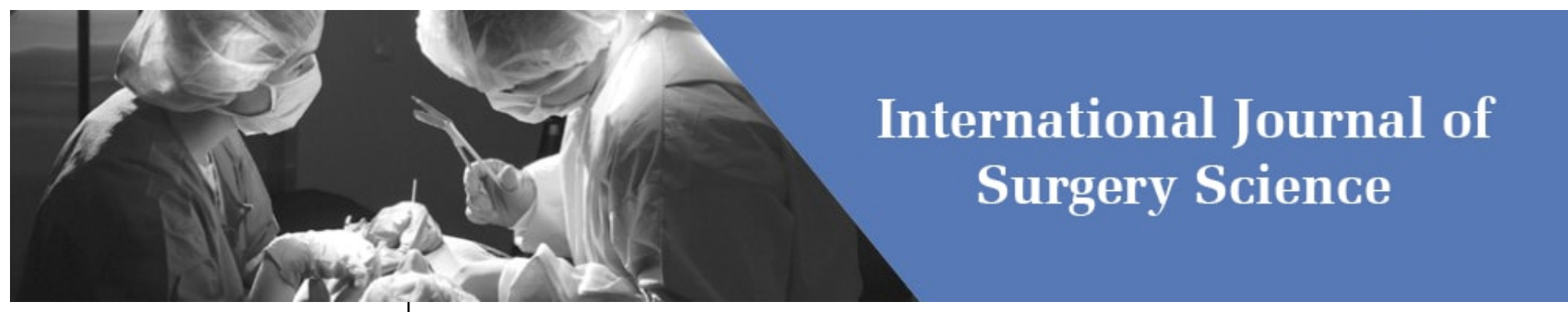

E-ISSN: 2616-3470

P-ISSN: 2616-3462

(C) Surgery Science www.surgeryscience.com

2020; 4(4): 157-163

Received: 19-08-2020

Accepted: 22-09-2020

Dr. Saad Shayaa

Al-Karkh Health Directorate,

Baghdad, Iraq
Corresponding Author:

Dr. Saad Shayaa

Al-Karkh Health Directorate,

Baghdad, Iraq

\section{Comparative study of operative fixation of fracture of medial malleolus by malleolar screw versus tension band wiring}

\section{Dr. Saad Shayaa}

DOI: https://doi.org/10.33545/surgery.2020.v4.i4c.553

\section{Abstract}

Background: Ankle fractures are common and many require surgical intervention. It has been well documented that type of surgery and a delay in fracture fixation results in increased length of hospital stay and increased complication rate. Initial delay can also allow swelling or blistering to develop which may necessitate a further delay in operative fixation up to 10 days and increase morbidity.

Patients and Methods: A prospective study of twenty-one patients presented with displaced closed fracture of medial malleolus and treated surgically at AL-KARKH Hospital from March 2010 to September 2011; the fractures had been fixed by tension-band wiring or by malleolar screw. The patients were then classified into two groups (15 patients in group 1 were treated by tension-band wiring, figure of eight, and 6 patients in group 2 were treated by malleolar screw fixation). The patients were evaluated clinically, radiologically, and functionally with follow-up till complete healing.

Results: Radiological union took place in the group 1 patients (mean time 10.2 weeks) earlier than in group 2 patients (mean time 12.2 weeks) with $\mathrm{p}=0.001$. Excellent and good functional results were achieved in $73 \%$ in group 1 patients and in $66.7 \%$ in group 2 patients with $p=0.034$. A better range-ofmotion (especially for dorsiflexion of ankle) was noticed in the group 2 patients.

Conclusions: Tension-band wiring may be more valid and excellent option than the malleolar screw for internal fixation of fracture of medial malleolus, as it is more stable fixation than malleolar screw, and allows early active exercises of the ankle and these results have to be supported by studies of larger number of cases and longer follow-up duration, and application of other methods of internal fixation, before recommending the optimum method for ankle fracture fixation.

Keywords: Fracture, fixation, ankle

\section{Introduction}

Because of the absence of radiography and the inchoate state of biomechanics, knowledge of ankle injuries prior to 1900 was based on clinical observations and cadaver experiments, but the significance of these early observations is clouded by ambiguous terminology, and by the failure of authors to distinguish experimental findings from clinical impressions ${ }^{[1]}$. The standard plain radiographic views of the injured ankle are the mortise view and lateral. The mortise view is a modified AP with the ankle internally rotated so that the malleoli are in the same horizontal plane and the joint space is seen evenly on both sides of the ankle. This requires 10-20deg. internal rotation. Adding, a true AP view does not add useful information and should not be part of the standard series. We obtain ankle Xrays weight bearing when possible, but in the acute trauma situation the patient usually cannot comply fully ${ }^{[39]}$. Lateral talar translation of more than 2mm is thought to imply a DTTL(distal tibiotalar ligament) tear and, therefore, instability However, medial clear space was a poor predictor of arthroscopically diagnosed deltoid injury. Some evidence favors' $4 \mathrm{~mm}$ as the maximum medial clear space compatible with deep deltoid competence; other evidence suggests $5 \mathrm{~mm}^{[38]}$.

Stress radiography: Stress radiograph used for ligament tear that not shown in plain x-ray. The above studies used a manually applied external rotation stress test. An alternative technique is the gravity stress test $50 \%$ had an abnormal stress test, although no additional imaging or clinical results were reported. 


\section{Recommendations [16, 38, 50].}

The assessment of the evidence on displacement and stability is: displacement is to be judged on talar position in the mortise, not fragment position either $4 \mathrm{~mm}$ or $5 \mathrm{~mm}$ of medial clear space would be justifiable as a starting point to define displacement, but $5 \mathrm{~mm}$ is attracting increasing supporting evidence medial signs are probably adequate to identify important deep deltoid injuries stress radiography probably overestimates the prevalence of important deep deltoid injuries prevalence of important deep deltoid injuries seems to lie between $1-20 \%$ of undisplaced fractures - as the highest estimate was in much the smallest study, the true value is probably in the lower part of this range. stress radiography is felt necessary, the gravity stress test may be more acceptable to patients than manual test.

Biomechanics of Tension band\& technique: Every eccentrically loaded bone is subjected to bending stresses. This results in a typical distribution of stresses with tension on the convex and compression on the concave side of bone. In order to restore the load - bearing capacity of an eccentrically loaded fracture bone, the tensile forces have to be absorbed by a tension band (wire, plate) and the bone itself has to be able to withstand axial compression ${ }^{[4]}$. The method essentially involves placing a wire loop dorsal to the midaxis of the eccentrically loaded fractured bone, thereby converting the destructive and shear forces tending to separate the fragments into compressive forces across the fracture site. This tension band will exert a force equal in magnitude but opposite in direction to the bending force [4]. The tension - band wire results in dynamic compression. The two kirschner wires are used to act as an internal splint neutralizing rotational and angular displacement forces. The compressive forces promote early fracture healing. This stable fixation also allows early mobilization and rehabilitation [4]. bone fragment is held in reduced position with tenaculum clamp; two $0.45 \mathrm{~K}$ wires are driven through the deltoid ligament and tip of medial malleolus and across fracture site but not into proximal tibial cortex; tension band figure of 8 wire (20 gauge) can be anchored proximally through an anterior to posterior drill hole in metaphysis (or by wrapping wire around head of the screw placed oblique in metaphysis) \& then passed around the $\mathrm{K}$ wires and tightened fashion (double twist technique is more reliable).

Biomechanics of lag screw: One of the most powerful and useful compression methods is that of the lag screw. Lag literally means "to follow". Any screw type may be used to produce a lag effect (squeeze) as long as three criteria are met: the screw in the near side fragment, threaded or nonthreaded, must glide freely in the near side screw hole (gliding hole) without engaging in the bone. Threads in the far side fragment screw must continue to progress through the bone as the screw turns and must firmly grip the bone without stripping. The advancement of the head of the screw must be solidly arrested on the surface of the near side ${ }^{[52,53,54]}$. Perfect re-alignment to reduce the fracture is essential, and the reduction is maintained by a bone clamp while a hole is drilled (by power drill) to receive the screw (gliding hole) in the near cortex, the drill diameter should be exactly equal to the core diameter of the screw, if it is wide; the screw thread will not engage and if it is narrow; too much force will be necessary to insert the screw and it may break, this is done in partially threaded screw with one drill bit, and during driving the lag screw into the hole, this allows the fracture fragments to be drawn together as the threaded part of the screw has been passing the fracture point.
Another method to produce such compression is by using a completed threaded screw with drilling the distal hole (gliding hole) in a diameter equal to the diameter of the thread, and drilling the proximal hole (thread hole) in a diameter equal to the diameter of the screw core. When the lag screw is used alone, the compression will be exerted along the screw and result in compression at the fracture interface; this is regarded as static interfragmental compression and it is optimum when the axis of the screw is perpendicular to the axis of the plane of the fracture. It is static because it does not change significantly with load; a lag screw is efficient way of achieving interfragmental compression and stability. It forms the basic building block of all stable fixations. It provides interfragmentary stability but does not provide a great deal of strength.

Conclusions: Threaded K-wires show substantial pullout strength and similar resistance to offset axial load when compared with partially threaded cancellous screws. These threaded K-wires offer an alternative for the internal fixation of medial malleolus fractures.

Fractures management principles: They can be divided into the following;
1. Undisplaced which can be further subdivided;
A. Stable
B. Unstable

\section{Displaced fracture}

Displacement refers to the position of the talus in the mortise, NOT minor displacements of the fibula. Fibular displacement in itself is of little significance. An intact deep deltoid ligament will keep the talus in the mortise in the face of fibular displacement. Apparent fibular displacements do not normally represent mortise incongruity. Residual mortise incongruity increases the risk of late osteoarthritis, although many patients with some incongruity or even OA have few symptoms. Therefore, the goal of treatment is to restore tibiotalar congruity, maintain this until fracture union and provide opportunities for rehabilitation. Even with optimum treatment, most patients will have some residual symptoms at long-term follow-up and they need to be advised of this before treatment begins. Initial management includes: management of other injuries, management of open wounds with cleansing, dressing, documentation and prophylaxis against pyogenic infection and tetanus, reduction of serious incongruity, especially if the medial skin is at risk, splintage, usually in a plaster back slab, pain relief, explanation, Traditionally, most displaced ankle fractures were managed by closed reduction and casting, with prolonged non weight bearing and follow-up radiography. Early internal fixation techniques emphasized stabilization of the medial malleolus with screws or k.wire \& tension band, believing that the lateral malleolus contributed little to ankle stability

\section{Pragmatic treatment, Cost Benefit with Early Operative} Fixation of Unstable Ankle Fractures:- It has been well documented that a delay in fracture fixation results in increased length of hospital stay and increased complication rate. Initial delay can also allow swelling or blistering to develop which may necessitate a further delay in operative fixation for up to 1 week. So early operative intervention for ankle fractures reduces the length of hospital stay, intensive physiotherapy and co-ordinate discharge planning are also essential ingredients for early discharge \& has substantial cost-saving implications with no 
increase in complication rate. The combined policies of early operative fixation and pro-active discharge planning minimize the financial implications of caring for this common fracture ${ }^{[24]}$.

\section{The influence of risk factors on the morbidity}

Ankle fractures and older patients: Ankle fractures in the elderly are associated with osteoporosis. They are more likely to have a stable configuration, especially in women and can be treated in the same way as undisplaced fractures in younger patients [57]. Displaced fractures require reduction and stabilization, as an alternative to plating the lateral malleolus, fibular nailing has been described ${ }^{[61]}$. Results were probably comparable with those of plating, possibly with fewer wound complications.

Ankle fractures and alcoholism: The postoperative morbidity after osteosynthesis of malleolar fractures was investigated retrospectively by comparing 10 alcohol abusers with 10 controls. The two groups were selected male patients and were matched regarding trauma, treatment for cardiovascular, pulmonary and endocrine diseases, age, weight, smoking habits, anaesthesia and duration of surgery. The alcohol abusers developed significantly more early complications, especially infections, after surgery. Follow-up at six, nine and 12 weeks after surgery also revealed a significantly higher morbidity among the alcoholics, added to that ankle fracture tend to occur at higher rate than others because of unstable gait in alcoholics.

Ankle fractures and Diabetics: The risks of treating ankle fractures in diabetics are higher than in nondiabetics whether surgical or closed methods are used ${ }^{[62]}$. Infection and skin breakdown are the main problems, and peripheral vascular disease, neuropathy and swelling increase the risk. The risk of wound failure after ORIF has been reported at 30-50\%. In open fractures the wound complication rate is $60 \%$ and the risk of amputation may be as high as $40 \%$ (White et al. 2003) ${ }^{\text {[63] }}$. In addition a fracture of the ankle may precipitate Charcot arthropathy in diabetics with peripheral neuropathy ${ }^{[64]}$. In highrisk neuropathic fractures transarticular fixation may give better stability than traditional osteosynthesis ${ }^{[65]}$. As in treating any foot and ankle problems in diabetics, it is extremely important to assess the severity and control of the diabetic condition and the presence of peripheral vascular disease, neuropathy, cardiac and renal failure. At the moment it seems reasonable to treat fractures in non-neuropathic patients on the same principles as the general population, but warning of increased risks, protecting for longer and monitoring for late displacement. In neuropathic patients we would consider treating displaced fractures with retrograde nails.

\section{Operative treatment}

Approaches to the medial malleolus: The patients are operated on under general anesthesia, patients are positioned supine, a sand-bag or a pillow below the affected ankle (according to surgeon's preference), and an Esmarch or Pneumatic tourniquet was applied to the lower limb. Preparation of the skin was done by povidone iodine $10 \%$, starting from below the site of tourniquet application and involving the toes.

1. Anteromedial approach; it offers an excellent view of medial malleolar fracture, and permits inspection of anteromedial ankle joint and anteromedial part of the dome of talus.

2. Posteromedial approach;it allows reduction and fixation of medial malleolar fracture and visualization of the posterior margin of the tibia.
Fixation of medial malleolus: Fixation of the medial malleolus usually consists of single or two malleolar screw $(4.5 \mathrm{~mm}$ malleolar or $4 \mathrm{~mm}$ partially threaded cancellous screws) oriented perpendicular to the fracture, vertical fracture of the medial malleolus require horizontally directly screw. Small fragment can be fixed with one malleolar screw or one malleolar screw and one kirschner wire or with two $1.6 \mathrm{~mm}$ or $2 \mathrm{~mm}$ smooth kirschner wire and a tension band. Fixation with a small semitubular buttress plate may be necessary to adequately stabilize vertical fracture that extends high into metaphysis.

After treatment: The ankle is immobilized in a posterior plaster splint with ankle in neutral position and elevated. If the bone quality is good and the fixation is secure, the splint can be removed in 2-4 days and replaced with a removable splint. Range of motion exercises is then begun. Weight - bearing is restricted for 6 weeks, after which partial weight bearing can be started if the fracture is healing well. Full weight bearing is allowed after 12 week. If poor skin condition, low bone quality, or insecure fixation, the fracture must be protected longer.

A wobble board: Is an excellent piece of exercise equipment that helps, enhance balance and ankle strength. If you have injured your ankle, you might find it difficult to regain strength and stability. Using a wobble board can aid rehabilitation or help prevent injuries.

1. Begin Slowly: Sit in a chair and place both feet on the wobble board about shoulder-width apart. Rotate the wobble board in all directions with your feet.

2. Rock the Wobble Board: Standing at the back of a chair, set the wobble board down and grasp the chair back for balance while you stand on the wobble board, your feet about shoulder-width apart. Rock on the wobble board back and forth and then from side to side. Do this slowly for about three minutes.

3. Rotate the Wobble Board While Standing Stand on the wobble board. Slowly rotate the board around and around so that the edges of the board come in contact with the floor. Do this for up to three minutes.

4. Balance on the Wobble Board: Eventually, as you gain more strength and balance, you will feel confident enough to stand on the wobble board. Try to stand on the board for at least three minutes while trying to keep the board still.

\section{Surgical Indications}

1. Fractures in which anatomic position of the ankle cannot be obtained or maintained by nonoperative methods. 2 . Displaced fractures of medial malleolus should be treated surgically because persistent displacement allows the talus to tilt into varus. 3. Most displaced bimalleolar fractures. 4. Nondisplaced Weber type c fractures are deceivingly innocent - looking but are often redisplace in cast, arguably, therefore, type c fracture are better fixed from outset. 5. Trimalleolar injuries: fractures of the posterior articular surface of the tibia require or if when more than $25 \%$ of the articular surface is involved. 6. Multiple injuries, 7. Patient in whom ORIF and early functional weight bearing management would be expected to convey an advantage.

\section{Complications}

- Immediate

- Fracture blisters

- Vascular injury.

- Early 
- Wound breakdown.

- Infection.

- Late

- Incomplete reduction.

- Non-union.

- Joint stiffness.

- Algodystrophy.

- Post-traumatic arthritis

The aim of the current study: To compare surgical fixation of medial malleolar fracture by malleolar screw and review the patient stay for operative ankle fractures over the previous 19month period at our hospital with the tension band wiring \& complication following the introduction of a fast-track system for the fixation of these fractures and find out the preferable method to achieve early mobilization and prevent contracture and stiffness with less complication and better healing rate.

Patient and Method: From March 2015 to September 2016 at Al-KARKH Hospital, we evaluate randomly 21 patients with displaced closed fracture of medial malleolus treated by open reduction and internal fixation with either malleolar screw or with tension-band wiring. The fracture were classified according to Danis-Weber classification and we exclude those with vertical fractures of medial malleolaus that's because these fracture require horizontally placed screws and also difficult to be fixed internally by tension-band wiring.

They were then classified into one of two groups:

- Group 1: which include 15 patients with tension-band wiring loop fixation method

- Group 2: which include 6 patients with a malleolar screw and washer fixation method. The surgery was performed within 48 hour.

\section{Fixation of the medial malleolus}

- Antibiotic therapy should begin immediately before surgery ( $\leq 2$ hours before incision and ideally 30 minutes before skin incision)

- Under general aneasthesia, patients were positioned supine, a sand-bag or a pillow below the affected ankle (according to surgeon's preference) and an Esmarch or pneumatic tourniquet was applied to the lower limb.

- Preparation of the skin done by povidone iodine $10 \%$ starting from below site of tourniquet application and involving the toes.

- We made an anteromedial incision that begins approximately 2cm proximal to the fracture line, extends distally and slightly posteriorly, and ends approximately $2 \mathrm{~cm}$ distal to the tip of medial malleolus. We prefer this incision for two reasons: first, the posterior tibial tendon and its sheath are less likely to be damaged, and second, the surgeon is able to see the articular surfaces, especially the anteromedial aspect of the joint, which permits accurate alignment of the fracture.

- $\quad$ The skin handled with care, reflecting the flap intact with its underlying subcutaneous tissues. The blood supply to the skin of this area is poor, and careful handling is necessary to prevent skin sloughing. Protect the greater saphenous vein and its accompying nerve.

- Usually the distal fragment of the medial malleolus is displaced distally and anteriorly, and a small fold of periosteum commonly is interposed between fracture surfaces, this fold should remove from the fracture site with a curette or periosteal elevator or even a knife, exposing the small serration of the fracture.

- Small, loose osseous or chondral fragments were debrided.

- With bone holding clamp or towel clip, we brought the detached malleolus into normal position, and while holding it there, internally fixed it with two 2-mm smooth $\mathrm{k}$. wires drilled across the fracture site \& the tension band wiring or malleolar screw is applied

- In group 1 patients, the medial malleolus drilled across the fracture and fixed with the two 2-mm smooth $\mathrm{k}$. wires perpendicular to the fracture line, the $\mathrm{k}$. wire should be parallel and their ends are bent at 90 degree angle; this will prevent the figure of eight wire from slipping over exposed ends of $\mathrm{k}$. wire, a hole is drilled in the proximal part of tibial fracture and then a stainless steel wire $1.2 \mathrm{~mm}$ AO passed through the previously drilled hole and around both bent ends of $\mathrm{k}$. wire in a figure of eight and then the wire tightened (especially if detached fragment was small or comminuted). In group 2 patients, a $3.2 \mathrm{~mm}$ hole was drilled in a superior posterior direction while distal segment was held reduced with pointed clamp or with $2 \mathrm{k}$. wires bent to stay out of way as temporary fixation device, length of the hole was measured, and malleolar screw was inserted without tapping till it reached the other cortex. K. wires were removed after screw was tightened. in 2(33.3\%) cases, the fragment were large and tend to rotate, so we used additional point of fixation (a second malleolar screw) The interior of the joint is carefully inspected, particularly at the superiomedial corner to insure the screw has not crossed the articular surface. Bulky hardware should be avoided to prevent wound complication of this poor coverage area. If both medial and lateral malleoli are fractured, we have to fix lateral malleolus first to restore length of fibula and then medial malleolus is fixed. Then integrity of the syndesmosis evaluated intra-operatively by grasping the intact or plated fibula with a hook or clamp and pulling it laterally, in 3 (14\%) cases out of 21, lateral displacement more than 3$4 \mathrm{~mm}$ occurred and therefore fixation by syndesmotic screw. At the end of operation, the tourniquet removed or deflated, good haemostasis obtained, and the wound closed, with interrupted suture (3/0 or 4 / nylon or silk). We avoided tight stitches to prevent necrosis of the skin edge; we applied thick sterilized padding and a posterior plaster splint with the ankle in neutral position.

\section{Postoperative management \&follow up}

Elevation of the foot until all swelling has resolved, administration of analgesia and the dressing reduced to well fit sterile gauze dressing followed by posterior plaster splint to prevent the foot from dropping into equines posture. After 48 hour, under supervision, active ankle movement within the comfortable range of motion were encourage several times daily, but the posterior plaster splint is re-applied between interval of treatment, at least comfortable active dorsiflexion above right angle was easily performed, at that stage, the backslab can be used only as night splint. Old patients may be referred with premorbid condition for physical therapist as they require formal physical therapy to successfully regain range of motion and strength. Once wound healing is assured (stitches should be removed after 10 to 14 day) and the wound examined and any complication was reported and treated accordingly, and progress is observed in regaining joint movement at 10 to 14 days, six weeks, three months and six months after operation. The patient may begin walking using crutches but without bearing weight 
and provided the fixation was regarded as stable, when there is any doubt about the stability of the fracture and patient compliance, a protective walking cast should be applied before discharge from hospital. Unprotected weight bearing is delayed until 6-8 weeks from operation, and where diastasis screw had been used, this should be removed first. The introduction of weight bearing will vary from patient to patient and will depend on the quality of the surgical fixation, local tissue factor and radiological control of the progress to bony union. Any appearance of the additional swelling, local warmth or redness would indicate the need to adopt a more cautious program of rehabilitation. In general, implants were removed from between 6 and 12 months from insertion.

The Results: The patients' data and correlated result are shown in the table;

Table 1: The profile of 21 patients with medial Malleolar fracture

\begin{tabular}{|c|c|c|}
\hline & $\begin{array}{c}\text { Group1 Tension band } \\
\text { wiring }\end{array}$ & $\begin{array}{c}\text { Group2 } \\
\text { Malleolar screw }\end{array}$ \\
\hline Mean age in years & $43.2 \pm 9.6$ & $41.5 \pm 17.6$ \\
\hline Male : Female ratio & $8: 7$ & $2: 4$ \\
\hline Right : Left ankle & $9: 6$ & $2: 4$ \\
\hline \multicolumn{3}{|c|}{ Causes of fracture } \\
\hline Fall from height & 5 & 1 \\
\hline Twisting & 4 & 2 \\
\hline Road traffic accident & 6 & 3 \\
\hline \multicolumn{3}{|c|}{ Fracture type } \\
\hline Transverse & 9 & 4 \\
\hline Oblique & 6 & 2 \\
\hline
\end{tabular}

Regarding age, gender, side, etiology or type of the fracture, there is no significant difference between two groups, table (2). Postoperative radiographs reveal anatomical reduction in both groups, in which the mean time for radiological bone union (indicated by disappearance of the fracture line) was about 10.2 weeks ranging from 6 to 13 weeks in group 1patients and 12.2 weeks ranging from 8 to 19 weeks in group 2 patients $(p=0.001)$ as in figure ${ }^{[33]}$. Stable fixation, normal fracture healing, and no patient had malunion, nonunion, or loss of reduction or signs of fixation failure or kirschner wires migration. Although better range of motion was noticed in group 2 patients (66\%) as compared with group1 (53\%), it improved later on with follow up and physiotherapy and so there is no limitation of movement or swelling and good range of joint motion in both groups.

\section{Discussion}

Broken ankles affect all ages, during the past few years there was an increase in the number and severity of broken ankles, due in part to road traffic accident, an active, older population of "baby boomers." In this study we are concerned about medial malleolar fracture, two different methods used for fixation either K.wire and tension band wiring or malleolar screw with variable results and we tried to find out which of those two methods result in better function and radiological outcome, so we put the patients into two groups; group (1) those are fixed with k. wire and tension band wiring (15 patient) and group (2) those are fixed with malleolar screw (6 patients) Tension band is more technically advantageous and it is stronger construct and stiffer over other types of fixation for fixation of avulsion fractures, osteoporotic bone fracture of medial malleolus ${ }^{[13,51,73]}$ that are too small to accept screw fixation. And Single screw fixation may provide poor stability against torsional force especially if fractured piece is large which may require additional fixation with malleolar screw. Limitation of ankle movement \& swelling in this study was 10 degree dorsiflexion \& 5 degree planter flexion in group 1 patients and non in group 2 patients and the former improved later with follow up good physiotherapy. Better range of motion was noticed in group 2 patients $(66 \%)$ as compared with group1 (53\%), this could be attributed to wide tissues dissection that was needed with use of tension band wiring. These results may be similar to the results of SKNurulAlam [70] who reported in his study that the group treated with malleolar screw showed better range of motion. No complication such as osteoarthritis was reported in this study which was assessed by radiologic finding of joint space narrowing, osteophyte formation and ligament calcification and this is because of stable anatomical reduction and no nonunion case. There is no significant difference between two groups regarding age, gender, side, etiology or type of the fracture, and probably due to small number of patients or short period of follow up.

\section{Conclusions}

1. The preliminary results of the current study suggest that tension-band wiring may be more valid option for internal fixation of medial malleolus fracture than malleolar screw fixation.

2. The tension-band wiring, in practice is more stable fixation \& technically advantageous for small and osteoporotic fragment fixation than malleolar screw, faster radiological union and allows early active exercises of the ankle.

3. Most complications that are related to the use of tensionband wiring fixation may be avoided by careful attention to the surgical technique (excessive pressure with bone clamps to hold the fracture reduction must be avoided to prevent crushing of the fragment particularly if the bone is osteoporotic. The fragment reduction instead can hold temporarily with kirschner wire and also excessive tighten and twisting of tension band wire to prevent cutting off).

4. Screw fixation alone may provide poor stability against torsion forces and may require an additional point of fixation which may be a second screw or kirschner wire.

5. Careful and meticulous soft tissue handling is important for prevention of postoperative wound complications, delayed union, and joint stiffness.

\section{Recommendations}

A study with larger number of patients with longer duration of follow up is needed to get precise results.

\section{Abbreviations}

(BKW) below knee walking, (MS) malleolar screw, (LS) lag screw, (LT) left, (RT) right

(TBW) tension band wire K.wire kirschner wire, (FIG) figure, (HR) hour, (DEG) degree,

(MUA) manipulation under anesthesia, (ORIF) open reduction internal fixation,

(MCS) medial clear space, (REF) reference, (RTA) road traffic accident, (Yr) year, (FFH)fall from hight.

\section{References}

1. Wilson FC, Skilbred LA. Long term results in the treatment of displaced fractures. J Bone Joint Surg Am 1966;48(6):1065-78.

2. Albert J, Kamra Aflaton D. Pathogenesis and treatment of ankle fractures. Journal of Southern Orthopaedic Association 2002. (Internet).web site; orhopaedic care.net. 
3. Wilson FC, Philip HO, Gilbert JA. Planter flexion injuries of ankle an experimental study clin ortho 1994;30(6):97102.

4. Solomon L, Warwich D, Nayagam S. Apley's system of orthopaedics and fracture. $8^{\text {th }}$ ed. London; Arnold, 2001, 733-744.

5. Michelson JD. Fracture about the ankle. J Bone J Surg Am 1995;77(1):142-149.

6. Pankovich A. Acute indirect ankle injuries in adult. J orthop Truama 2002;16(1):58-68.

7. Richard S. Snell Clinical anatomy for medical student. $5^{\text {th }}$ ed. USA; mosby Company 1998, 658-661.

8. Pankovich A, Shiviram MS. Anatomical basis of variability in injuries of the medial malleolus and deltoid ligament. Acta orthop Scand 1979;50:225.

9. Gonza ER, Harrington IJ. Biomechanics of musculoskeletal injuries. $1^{\text {st }}$ ed Baltimore: William and wilkin 1982, 1-25, 87-129.

10. Burstein AH, Frankel VH. Biomechanics of the ankle. Orthop Clinic. North America 1973;96:323-326.

11. Michelson JD, Ahn UM. Dynamic biomechanics of the normal and fractured ankle. Trans orhop Res Soc 1994;40:253.

12. Muller ME, Allgower M, Schnieder R, et al. Manual of internal fixation. $3^{\text {rd }}$ Ed. New York: Springer-Verlag 1991, 27, 42, 248.

13. Ramsey PL, Hamilton W. Changes in tibiotalar contact area caused by lateral talar shift. JBJS 1976;58A:356-357.

14. Pereira DS, et al. Tibiotalar contact area and pressure distribution: the effect of mortise widening and syndesmosis fixation. FAI 1996;17:269-74

15. Lloyd J, et al. Revisiting the concept of talar shift in ankle fractures. FAI 2006;27:793-6.

16. http:foothyperbook.com/trauma/malleolarfx/anklefxbasic4.h tm06/02/2011 [12:05:20Am]

17. Lauge-Hansen N. Fractures of the ankle II. Combined experimental-surgical and experimental-roentgenologic investigations. Arch Surg 1950;60:957-85.

18. Yde J, Kristensen KD. Supination-eversion fractures of stage 2. Acta Orthopaedica Scandinavia 1980;51:103-106.

19. Yde J, Kristensen K. Ankle fractures: supination-eversion fractures of stage IV. AOS 1980;51:981-9

20. Gardner MJ, et al. The ability of the Lauge-Hansen classification to predict ligament injury and mechanism in ankle fractures: an MRI study. J Orthop Trauma 2006;20:267-72.

21. Kennedy JG, Soffe KE, Dalla Vedova P, Stephens MM, O'Brien T, Walsh MG, et al. Evaluation of the syndesmotic screw in low Weber C ankle fractures, JOT 2000;14:359-66.

22. Weber B. Ankle fracture: an unnecessary fracture clinic burden. Injury 2004;35:805-8.

23. Michelson JD, et al. Examination of the pathological anatomy of ankle fractures. J. Trauma 1992;32:65-70.

24. Michelson JD, et al. Motion of the ankle in a simulated supination- external rotation fracture model. JBJS 1996;78A:1024-1031.

25. Michelson JD, et al. Economic analysis of roentgenogram use in the closed treatment of stable ankle fractures. J Trauma 1995;39:1119-1122.

26. Http://foothyperbook.com/trauma/malleolarFx/ankleFxMgt MedMal1.htm[05/02/2011 [11:42:10 pm]

27. Rockwood CA, Green DP, Heckmann JD. Fractures in adults. $4^{\text {th }}$ ed. Philadelphia: Lippincott-Raven 1996:22192222.
28. Rovinisky D, et al. Evaluation of a new method of small fragment in a medial malleolar fracture. J Orthopaedics Trauma 2000;14(6):420-425.

29. McConnell $\mathrm{T}$, et al. Stress examination of supination external rotation-type fibular fractures. JBJS 2004;86A:2171-8.

30. Kim SK, et al. One or Two lag screws for fixation of Danis Weber type $B$ fractures of the ankle. J Trauma 1999;46(6):1039-1044.

31. Haraguchi N. Pathoanatomy of posterior malleolar fractures of the ankle. JBJS 2006;88A:1085-92.

32. Burns WC, et al. Tibiotalar joint dynamics: indications for the syndesmotic screw--a cadaver study. Foot Ankle 1993;14:153-8.

33. http://foothyperbook.com/trauma/malleolarFx/ankleFxBasic 2.htm[05/02/2011[ 11:45:13pm]

34. http://foothyperbook.com/trauma/malleolarFx/ankleFxClini cal1.htm[06/02/2011 [12:02:28Pm]

35. Laarhoven CJ, Meeuwis J, et al. Postoperative treatment of internally fixed ankle fractures. J Bone Joint Surg 1996;78(3):395-9.

36. Stiell I, et al. Multicentre trial to introduce the Ottawa ankle rules for use of radiography in acute ankle injuries. Multicentre Ankle Rule Study Group. BMJ 1995;311(7005):594-7.

37. http://foothyperbook.com/trauma/malleolarFx/ankleFxImag es. htm [06/02/2011 [12:04:02am]

38. Vangsness CT, et al. Radiographic diagnosis of ankle fractures:are three views necessary? FAI 1994;15:172-4.

39. Schwartz DT, Reisdorff E, Williamson B, et al. Emergency radiology. $1^{\text {st }}$ ed. New York: McGraw-Hill 1999, 166-68.

40. Brage ME, et al. Observer reliability in ankle radiographic measurements. FAI 1997;18:324-9.

41. Tang CW, et al. Position of the distal fibular fragment in pronation and supination ankle fractures: a CT evaluation. Foot Ankle Int, 2003.

42. Weber $\mathrm{M}$, et al. The use of weightbearing radiographs to assess the stability of supination-external rotation fractures of the ankle. Arch Orthop Trauma Surg 2010;130(5):693-8.

43. McConnell $\mathrm{T}$, et al. Stress examination of supination external rotation-type fibular fractures. JBJS 2004;86A:2171-8.

44. Egol KA, et al. Ankle stress test for predicting the need for surgical fixation of isolated fibular fractures. JBJS 2004;86A:2392-8.

45. Michelson JD. Fractures around the ankle. JBJS 1995;77A(1):142-149.

46. Schock HJ, et al. The use of gravity or manual-stress radiographs in the assessment of supination-external rotation fractures of the ankle. JBJS 2006;89B:1055-9.

47. Weber $\mathrm{M}$, et al. The use of weightbearing radiographs to assess the stability of supination-external rotation fractures of the ankle. Arch Orthop Trauma Surg 2010;130(5):693-8.

48. Koval KJ, et al. Does a positive ankle stress test indicate the need for operative treatment after lateral malleolus fracture? A preliminary report. JOrthop Trauma 2007;21:449-55.

49. Xenos JS, et al. The tibiofibular syndesmosis. Evaluation of the ligamentous structures, methods of fixation, and radiographic assessment. JBJS 1995;77A:847-56.

50. Tension band fixation of medial malleolus fractures. RF Ostrum and AS Litsky J. Orthop. Trauma 1992;6(4):464468.

51. JN Wilson. Watson-Jones fractures and joint injuries. $6^{\text {th }}$ Ed.Churchill Livingstone 1986;1:374-77. 
52. Rockwood C.A, Green D.; Fractures in adults. Rockwood And Green's, $4^{\text {th }}$ ed. Lippincott-Raven 1996, 167-171.

53. Müller ME, Allgöwer M. Manual of internal fixation Springer-Verlag Berlin, $3^{\text {rd }}$ edition 1995, 188-190, 226-228.

54. http://foothyperbook.com/trauma/malleolarFx/ankleFxMgtI ntro1.htm [06/02/2011 12:05:20 am]

55. Yablon IG, Heller FG, LeRoy S. The key role of the lateral malleolus in displaced fractures of the ankle. JBJS 1977;59A(2):p169-173.

56. Fox A, et al. Five years of ankle fractures grouped by stability. Injury 2005;36:836-41.

57. Akhtar S et al. Pragmatic treatment of ankle fractures of uncertain stability: clinical features and risk of displacement. BOFAS, 2009.

58. Makwana NK, et al. Conservative versus operative treatment for displaced ankle fractures in patients over 55 years of age. A prospective, randomized study. JBJS 2001;83B:525-9.

59. Srinivasan CM, Moran CG. Internal fixation of ankle fractures in the very elderly. Injury 2001;32(7):559-63.

60. Gehr J, et al. New concepts in the treatment of ankle joint fractures. The IPXS (XSL) and IP-XXS (XXSL) nail in the treatment of ankle joint fractures.Arch Orthop Trauma Surg 2004;124(2):96-103.

61. Flynn JM, et al. Closed ankle fractures in the diabetic patient. Foot Ankle Int 2000;21(4):311-9.

62. White CB, et al. Open ankle fractures in patients with diabetes mellitus. Clin Orthop 2003;(414):37-44.

63. Kristiansen B. Ankle and foot fractures in diabetics provoking neuropathic joint changes. Acta Orthop Scand 1980;51(6):975-9.

64. Jani MM, et al. A protocol for treatment of unstable ankle fractures using transarticular fixation in patients with diabetes mellitus and loss of protective sensibility. Foot Ankle Int 2003;24(11):838-44.

65. Wobble Board Exercises ;Balance Boards and Ankle Exercises, Read more: Wobble Board Exercise for Ankles | eHow.com http:/www.ehow.com/way_5250252_wobbleboard-exercise-ankles.html\#ixzz1cXhw6fOj

66. From Johnson EE, Davlin LB. Open ankle fractures: the indications for immediate open reduction and internal fixation, ClinOrthopRelat Res 1993;292:118.

67. Sang-Hanko M, Young-JunPark D. Comparison between screw fixation and tension -band wiring for medial malleolus fractures. J Korean Society of Foot Surg 2002;6(1):41-44.

68. Nurul SK, Lam A, Parviz Shahidi, et al. Comparative study of malleolar fractures by tension band and malleolar screw. BOS Journal 1997;12(1):13-19.

69. Mack D, Szabo RM. Complications of tension band wiring. J Bone Joint Surg 1985;67:1936-1941.

70. Kinik H, Mergen E. Self-locking tension band technique. Arch Orthop Truama Surg 1999;119:432-434.

71. Finsen V, Saetermo R, et al. Early postoperative weightbearing and muscle activity in patients who have a fracture of the ankle. J Bone Joint Surg 1989, 71-23.

72. Jones, Peter S. Single screw used for medial malleolar ankle fractures. AAOS On-line Service Academy News-Section B. Today's News. Saturday, (internet), 1997. 